Proceedings

\title{
Allium cepa L. inoculation with a consortium of plant growth-promoting bacteria: effects on plant growth and development and soil fertility status and microbial community ${ }^{\dagger}$
}

\author{
Marika Pellegrini 1,2,*, Daniela Spera ${ }^{1}$, Claudia Ercole ${ }^{2}$, and Maddalena del Gallo ${ }^{2}$ \\ 1 AGIRE Soc. cons. a r.l., Via Isidoro e Lepido Facii - 64100 Teramo, Italy; m.pelegrini@poloagire.it (M.P.); \\ danielamspera@gmail.com (D.M.S.) \\ 2 University of L'Aquila, Dep. MeSVA, Via Vetoio - 67100 L'Aquila, Italy; claudia.ercole@univaq.it (C.E.); \\ maddalena.delgallo@univaq.it (M.D.G.) \\ * Correspondence: marika.pellegrini@guest.univaq.it; Tel.: +39-0862-433246 \\ + Presented at the 1st International Electronic Conference on Microbiology, 2-30 November 2020; Available \\ online: https://ecm2020.sciforum.net/
}

Published: 2 November 2020

\begin{abstract}
The present work was aimed at investigating the effects of a four strains consortium Azospirillum brasilense, Gluconacetobacter diazotrophicus, Herbaspirillum seropedicae, and Burkholderia ambifaria - on crops of Allium cepa L. and its soil health. The bacterial consortium was inoculated on seeds of two different onion varieties; inoculated seeds and control ones (treated with autoclaved inoculum) were sown in open-field and followed until harvest. Plant growth development parameters, as well as soil physico-chemical and molecular profiles (DNA extraction and 16S community sequencing on the Mi-Seq Illumina platform), were investigated. The results showed a positive influence of bacterial application on plant growth, with increased plant height $(+18 \%)$, total chlorophylls $(+42 \%)$, crop yields $(+13 \%)$ and bulbs dry matter $(+3 \%)$ than the control. The differences between control and treated experimental conditions were also underlined in the bulb extracts in terms of total phenolic contents ( $+25 \%)$ and antioxidant activities $(+20 \%)$. Soil fertility and microbial community structure and diversity were also positively affected by the bacterial inoculum. At harvest, the soil with the presence of the bacterial consortium showed an increase of total organic carbon, organic matter and available $\mathrm{P}$ and higher concentrations of nutrients than control. The ecological indexes calculated on the molecular profiles showed that community diversity was positively affected by the bacterial treatment. The present work allowed to remark the effective use of plant growth-promoting bacteria as valid fertilization strategy to improve yield in productive landscapes, whilst safeguarding soil biodiversity.
\end{abstract}

Keywords: Biostimulants; PGPB; Seed inoculation; Sustainable agriculture; Illumina sequencing

\section{Introduction}

One of the current concerns in agriculture is improving the sustainability of productive landscapes and at the same time obtaining high production rates. To achieve this goal one of the promising and sustainable innovation could be the use of natural plant biostimulants [1]. Among them plant growth-promoting rhizobacteria (PGPR) are considered sustainable biostimulant agents, effective also in the presence of abiotic stresses factors [1]. Key traits for biostimulation are nitrogen fixation, nutrients solubilizing capabilities and production of phytohormones [2]. During the past decades, PGPB formulations gained increasing attention, being highly regulated from European Union by Regulation EU 2019/1009. Seed inoculation is the most common method of application. In 
this work we investigated the biostimulant effectiveness of a seed inoculation with a bacterial consortium formed by Azospirillum brasilense Cd, Burkholderia ambifaria PHP7, Gluconacetobacter diazotrophicus Pal5 and Herbaspirillum seropedicae Z67. The consortium of these strains already demonstrated good biostimulant effects on Lycopersicon esculentum L. [3] Cannabis sativa L. [4] Artemisia eriantha Ten [5] and ancient Triticum genotypes [6].

In this work, we hypothesized that this PGPR consortium could positively affect growth, development, yield, and quality traits of Allium cepa crops. To demonstrate the validity of this hypothesis, two onion varieties were chosen ("Meranto" and "Moondance"). Firstly, the bacterial adhesion on seeds were assessed by SEM. Then, two parallel one-year field experiments were carried out to evaluate the effectiveness of seed PGPR treatment on plants (i.e. height and chlorophylls amount) and bulbs (i.e. dry weight, total phenolic content, antioxidant activity). To evaluate the influence of this treatment on soil structure and fertility status, crop field samples were investigated for their physico-chemical and molecular profiles (DNA extraction and NGS sequencing).

\section{Material and Methods}

The bacterial consortium was prepared as previously described by Botta et al. (2013). Onion seeds (var. Moondance and Meranto) were treated with the bacterial consortium and dried overnight at room temperature. Controls were treated in the same way, utilizing autoclaved inoculum. Sowings were performed by automatic seeder following a split-plot arranged experimental design. During the cultivations, plant height and chlorophylls [7] were monitored. At harvest, yields were quantified and bulbs were extracted [8] and investigated for their total phenolic content (by Folin-Ciocâlteu reagent) and antioxidant activity (by DPPH, ABTS and FRAP assays) [9].

Soil physico-chemical analyses were carried out according to the official methods described in “DM 13/09/1999 GU N²48 21/10/1999”. Genomic DNA was extracted utilizing the NucleoSpin $®$ Soil kit (Macherey Nagel, Germany), following the manufacturer's protocol. DNA samples were amplified by a specific $16 \mathrm{~S}$ protocol for the amplification of bacteria and archea, using paired-end $16 \mathrm{~S}$ community sequencing on the Mi-Seq Illumina platform (Bio-Fab Research, Italy). The 16S V3 and V4 region were targeted by gene-specific sequences [10]. Classifications were obtained through a metagenomics workflow on Greengenes database (http://greengenes.lbl.gov). Data were processed by QIIME2 and Diversity indices were calculated using the $\mathrm{R}$ ( $\mathrm{R}$ Foundation for Statistical Computing, Vienna, Austria) statistical package vegan v2.5-665.

\section{Results and Discussion}

In Figure 1 are shown the micrographs obtained by SEM, in which is clear that bacterial strains were able to adhere to seed surfaces. The bacterial adhesion occurs thanks to biofilm formation, a basic characteristic generally attributed to strains belonging to the PGPB and key element for their association with plants [11].
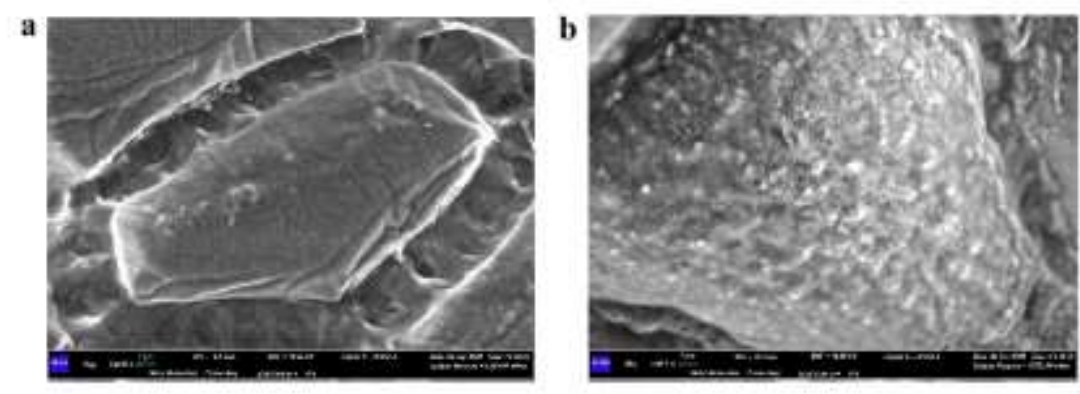

Figure 1. SEM micrographs at 5000X of (a) control and (b) inoculated Allium cepa seed.

The presence of PGPB promoted best growth and development of both plants and bulbs than control (Figure 2). On average, were recorded increases in terms of: (i) plant height, $18 \%(p<0.001)$; (ii) total chlorophylls, $42 \%$ ( $\mathrm{p}<0.001$ ); (iii) crop yield, $13 \%$ ( $\mathrm{p}<0.01$ ); (iv) bulbs dry matter, $3 \%(\mathrm{p}<0.05)$. 
Total phenolic contents and antioxidant activities of bulbs extracts also recorded positive increases $25 \%(\mathrm{p}<0.01)$ and $20 \%(\mathrm{p}<0.05)$, respectively. PGPB biostimulants properties are well-known and can be ascribed to several direct and indirect mechanisms [12]. The capability of synthesizing hormones, stimulates plant development and have positive influence on pigments synthesis [13]. The capability of solubilizing nutrients (e.g. P and K) allows plants to receive nourishment and helps them to thrive. Crop yield and bioactive compounds increases are other positive consequences of biostimulants effects.

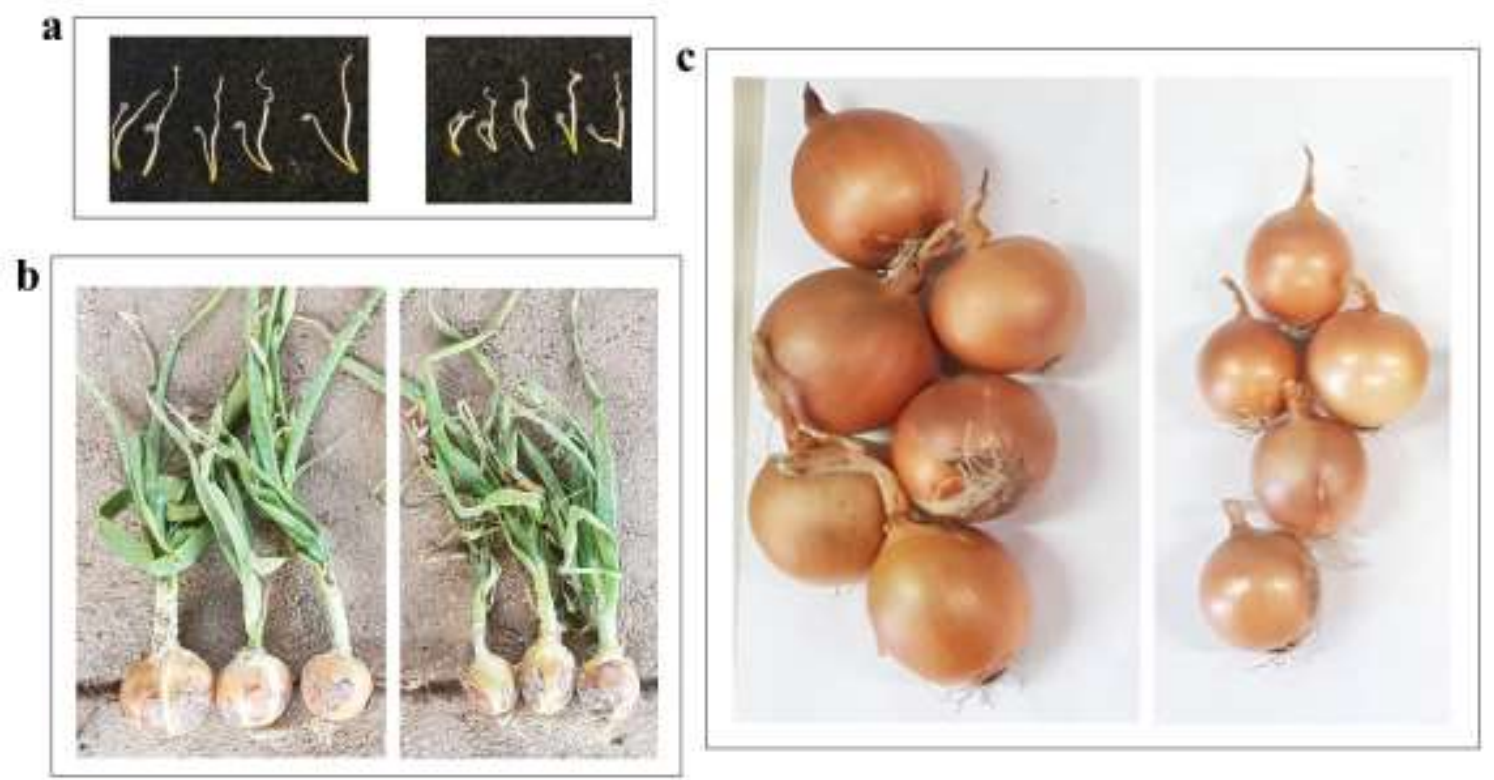

Figure 2. (a) Seedling, (b) plants and (c) bulbs of Allium cepa. In the panels, inoculated plants are on the left, while controls are on the right.

PGPB positive influence was shown also in terms of soil fertility status. In both experimental fields, the application of PGPB consortium increased total organic carbon, organic matter and available $P$ and allowed to keep higher concentrations of the other nutrients. These findings have been already reported by other authors $[14,15]$ and can be ascribed to the PGPB capability of nutrient mobilization and to atmospheric nitrogen fixation [2]. The ecological indexes calculated on NGS results showed that seed bacterization was able to positively influence soil microbial community composition of both varieties, by increasing microbial biodiversity. The PGPB influence on soil microbial community has been underlined also by other authors [16], however, the mechanisms behind it have not been yet completely clarified [17].

\section{Conclusions}

Our results underlined that the onion seed treatment with a selected PGPB consortium is an effective methodology that can significantly improve yield and quality of onion crops. Further studies should be undertaken to assess the biostimulant ability on different soils and environmental conditions. Anyhow, the results so far obtained suggest that increasing bacterial biodiversity could be a valid eco-friendly technique to increase food quality whilst safeguarding soil biodiversity.

Author Contributions: Conceptualization, M.D.G. and D.S.; methodology, C.E.; formal analysis, M.P.; investigation, M.P.; data curation, M.P.; writing-original draft preparation, M.P. and D.S.; writing-review and editing, M.D.G.; supervision, M.D.G.; project administration, D.S.

Funding: This research was funded by “Programma Operativo Regionale (POR-FESR) - Azione 1.1.1 Progetti di Ricerca Industriale e Sviluppo Sperimentale delle Imprese afferenti ai Domini individuati nella RIS3 della Regione Abruzzo - INNOPAQ CUP C43D18000130007". 
Acknowledgments: We wish to thank "Azienda Agricola Scipioni" for the provision and for the cultivation of experimental fields and Lorenzo Arrizza for the support in the Scanning Electron Microscope analysis.

Conflicts of Interest: The authors declare no conflict of interest. The funders had no role in the design of the study; in the collection, analyses, or interpretation of data; in the writing of the manuscript, or in the decision to publish the results.

\section{References}

1. Colla, G.; Rouphael, Y.; Canaguier, R.; Svecova, E.; Cardarelli, M. Biostimulant action of a plant-derived protein hydrolysate produced through enzymatic hydrolysis. 2014, 5, 1-6, doi:10.3389/fpls.2014.00448.

2. Bashan, Y.; de-Bashan, L.E.; Prabhu, S.R.; Hernandez, J.P. Advances in plant growth-promoting bacterial inoculant technology: Formulations and practical perspectives (1998-2013). Plant Soil 2014, 378, 1-33, doi:10.1007/s11104-013-1956-x.

3. Botta, A.L.; Santacecilia, A.; Ercole, C.; Cacchio, P.; Del Gallo, M. In vitro and in vivo inoculation of four endophytic bacteria on Lycopersicon esculentum. N. Biotechnol. 2013, 30, 666-674, doi:10.1016/j.nbt.2013.01.001.

4. Pagnani, G.; Pellegrini, M.; Galieni, A.; Egidio, S.D.; Matteucci, F.; Ricci, A.; Stagnari, F.; Sergi, M.; Lo, C.; Pisante, M.; et al. Plant growth-promoting rhizobacteria (PGPR) in Cannabis sativa 'Finola' cultivation: An alternative fertilization strategy to improve plant growth and quality characteristics. Ind. Crop. Prod. 2018, 123, 75-83, doi:10.1016/j.indcrop.2018.06.033.

5. Pace, L.; Pellegrini, M.; Palmieri, S.; Rocchi, R.; Lippa, L.; Del Gallo, M. Plant growth-promoting rhizobacteria for in vitro and ex vitro performance enhancement of Apennines' Genepì (Artemisia umbelliformis subsp. eriantha), an endangered phytotherapeutic plant. Vitr. Cell. Dev. Biol. - Plant 2020, 56, 134-142, doi:10.1007/s11627-019-10035-1.

6. Pagnani, G.; Galieni, A.; Stagnari, F.; Pellegrini, M.; Gallo, M. Del; Pisante, M. Open field inoculation with PGPR as a strategy to manage fertilization of ancient Triticum genotypes. Biol. Fertil. Soils 2020, 56, 111-124, doi:10.1007/s00374-019-01407-1.

7. Arnon, D.I. Copper enzymes in isolated chloroplasts. Polyphenoloxidase in Beta vulgaris. Plant Physiol. 1949, 24, 1-15, doi:10.1104/pp.900074.

8. Mirzapour, M.; Hamedi, M.; Rahimipanah, M. Sunflower Oil Stabilization by Persian Walnut Leaves Extract during Oven Storage Test. Food Sci. Technol. Res. 2010, 16, 443-446.

9. Pellegrini, M.; Id, A.R.; Id, A.S.; Chaves-1, C. Characterization of Essential Oils Obtained from Abruzzo Autochthonous Plants: Antioxidant and Antimicrobial Activities Assessment for Food Application. Foods 2018, 7, 19, doi:10.3390/foods7020019.

10. Klindworth, A.; Pruesse, E.; Schweer, T.; Peplies, J.; Quast, C.; Horn, M.; Glöckner, F.O. Evaluation of general $16 \mathrm{~S}$ ribosomal RNA gene PCR primers for classical and next-generation sequencing-based diversity studies. Nucleic Acids Res. 2013, 41, e1-e1, doi:10.1093/nar/gks808.

11. Djebaili, R.; Pellegrini, M.; Smati, M.; Del Gallo, M.; Kitouni, M. Actinomycete Strains Isolated from Saline Soils: Plant-Growth-Promoting Traits and Inoculation Effects on Solanum lycopersicum. Sustainability 2020, 12, 4617, doi:10.3390/su12114617.

12. Vejan, P.; Abdullah, R.; Khadiran, T.; Ismail, S.; Nasrulhaq Boyce, A. Role of plant growth promoting rhizobacteria in agricultural sustainability-A review. Molecules 2016, 21, 1-17, doi:10.3390/molecules21050573.

13. Urana, R.; Singh, N.; Sharma, P. Effects of PGPR on growth and photosynthetic pigment of Trigonella foenum - graceum and Brassica juncea in PAH - contaminated soil. SN Appl. Sci. 2019, 1, 1-9, doi:10.1007/s42452-0190780-1.

14. Orhan, E.; Esitken, A.; Ercisli, S.; Turan, M.; Sahin, F. Effects of plant growth promoting rhizobacteria (PGPR) on yield, growth and nutrient contents in organically growing raspberry. Sci. Hortic. (Amsterdam). 2006, 111, 38-43, doi:10.1016/j.scienta.2006.09.002.

15. Ullah, A.; Bano, A. Role of PGPR in the reclamation and revegetation of saline land. Pakistan J. Bot. 2019, 51, 27-35, doi:10.30848/PJB2019-1(43).

16. Hou, J.; Liu, W.; Wang, B.; Wang, Q.; Luo, Y.; Franks, A.E. PGPR enhanced phytoremediation of petroleum contaminated soil and rhizosphere microbial community response. Chemosphere 2015, 138, 592-598, doi:10.1016/j.chemosphere.2015.07.025. 
17. Ren, H.; Huang, B.; Fernández-García, V.; Miesel, J.; Yan, L.; Lv, C. Biochar and rhizobacteria amendments improve several soil properties and bacterial diversity. Microorganisms 2020, 8, 502, doi:10.3390/microorganisms8040502.

Publisher's Note: MDPI stays neutral with regard to jurisdictional claims in published maps and institutional affiliations.

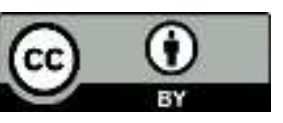

(C) 2020 by the authors. Submitted for possible open access publication under the terms and conditions of the Creative Commons Attribution (CC BY) license (http://creativecommons.org/licenses/by/4.0/). 